\title{
Corrigendum
}

\section{Computation of collective modes and acoustic investigations at different temperatures of vitrous silica}

R V GOPALA RAO and R VENKATESH

(Pramana - J. Phys., 43, pp. 201-209, September 1994)

The following acknowledgement was inadvertently omitted from the paper:

"One of us (RVGR) acknowledges the support of INSA, New Delhi, through a Senior Scientist position." 